\title{
Local connectivity effects in learning and coordination dynamics in a two-layer network
}

Cite as: Chaos 30, 083125 (2020); https://doi.org/10.1063/5.0006908

Submitted: 09 March 2020 . Accepted: 27 July 2020 . Published Online: 11 August 2020

Haydée Lugo (D), Juan Carlos González-Avella, and Maxi San Miguel (D)

\section{COLLECTIONS}

Paper published as part of the special topic on Instabilities and Nonequilibrium Structures Note: This article is part of the Focus Issue, instabilities and Nonequilibrium Structures.
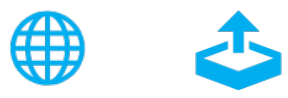

\section{ARTICLES YOU MAY BE INTERESTED IN}

\section{Cooperation delay induced chaos in an ecological system}

Chaos: An Interdisciplinary Journal of Nonlinear Science 30, 083124 (2020); https:// doi.org/10.1063/5.0012880

A generalized linear threshold model for an improved description of the spreading dynamics

Chaos: An Interdisciplinary Journal of Nonlinear Science 30, 083127 (2020); https:// doi.org/10.1063/5.0011658

Spreading of two interacting diseases in multiplex networks

Chaos: An Interdisciplinary Journal of Nonlinear Science 30, 073115 (2020); https:// doi.org/10.1063/5.0009588

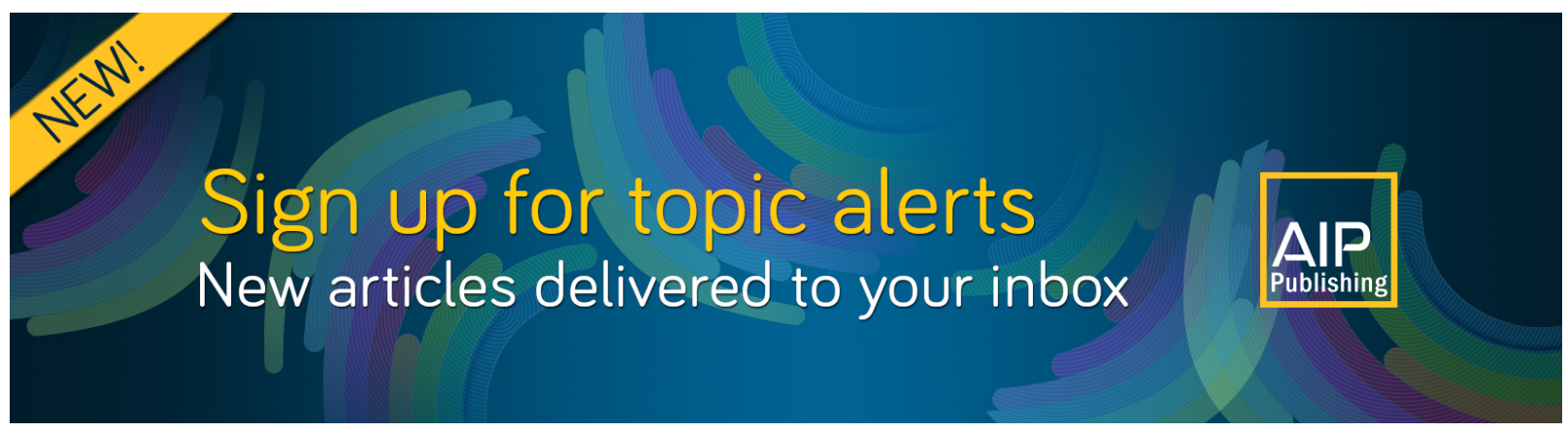




\title{
Local connectivity effects in learning and coordination dynamics in a two-layer network
}

\author{
Cite as: Chaos 30, 083125 (2020); doi: $10.1063 / 5.0006908$ \\ Submitted: 9 March 2020 . Accepted: 27 July 2020 . \\ Published Online: 11 August 2020

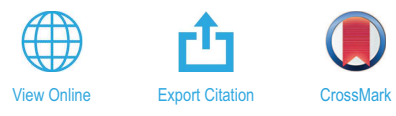

Haydée Lugo, , ,a) (D Juan Carlos González-Avella, ${ }^{2, b)}$ and Maxi San Miguel ${ }^{3}$ (D)

\begin{abstract}
AFFILIATIONS
${ }^{1}$ ICAE and Department of Economic Analysis, Universidad Complutense de Madrid, 28223 Madrid, Spain

${ }^{2}$ APSL S.L, Edifici Europa_Planta baja Galileo Galilei, Parc Bit, 07121 Palma de Mallorca, Spain

${ }^{3}$ IFISC (CSIC-UIB), Campus Universitat de les IIles Balears, 07122 Palma de Mallorca, Spain
\end{abstract}

Note: This article is part of the Focus Issue, instabilities and Nonequilibrium Structures.

a) Author to whom correspondence should be addressed: hlugo@ucm.es

Also at: IFISC (CSIC-UIB), Campus Universitat de les IIles Balears, 07122 Palma de Mallorca, Spain.

\begin{abstract}
Anticoordination and chimera states occur in a two-layer model of learning and coordination dynamics in fully connected networks. Learning occurs in the intra-layer networks, while a coordination game is played in the inter-layer network. In this paper, we study the robustness of these states against local effects introduced by the local connectivity of random networks. We identify threshold values for the mean degree of the networks such that below these values, local effects prevent the existence of anticoordination and chimera states found in the fully connected setting. Local effects in the intra-layer learning network are more important than in the inter-layer network in preventing the existence of anticoordination states. The local connectivity of the intra- and inter-layer networks is important to avoid the occurrence of chimera states, but the local effects are stronger in the inter-layer coordination network than in the intra-layer learning network. We also study the effect of local connectivity on the problem of equilibrium selection in the asymmetric coordination game, showing that local effects favor the selection of the Pareto-dominant equilibrium in situations in which the risk-dominant equilibrium is selected in the fully connected network. In this case, again, the important local effects are those associated with the coordination dynamics inter-layer network. Indeed, lower average degree of the network connectivity between layers reduces the probability of achieving the risk-dominant strategy, favoring the Pareto-dominant equilibrium.
\end{abstract}

Published under license by AIP Publishing. https://doi.org/10.1063/5.0006908

The game-theoretical model of coordination illustrates the problem of equilibrium selection. Collective dynamics in social systems embraces a rich phenomenology of nonequilibrium structures and instabilities. Among these structures are anticoordination states where two parts of a system coexist in two absorbing states corresponding to a different consensus among two different possibilities and also chimera states where one part of the system reaches a consensus absorbing state, while the other part remains in a dynamical state. These two nonequilibrium structures occur in a two-layer network model with two simple dynamics of interaction: intra-layer dynamics of social learning and inter-layer coordination dynamics in the framework of evolutionary game theory. In this paper, we discuss the instability of these structures as a consequence of local effects: They become unstable below a threshold value of a parameter measuring local connectivity of the network. In addition, we discuss the effects of locality in the question of equilibrium selection (selection of two different possible asymptotic states) in the coordination game.

\section{INTRODUCTION}

In real-life situations, it is common to observe the lack of a common opinion or strategy (consensus) among individuals. In many cases, this is due to the existence of multiple desirable outcomes or to the existence of a risky efficient outcome. However, it may also be due to competing effects of strategic learning and of social learning under the influence of social pressure. With this motivation, we introduced in Ref. 1 a dynamical model of social coordination through a two-layer network considering a 
population with two different types of interactions: (1) an interlayer strategic interaction through a coordination game ${ }^{2}$ and (2) an intra-layer social interaction where agents are influenced by their peers. ${ }^{3,4}$ Within this model, it has been shown that when agents are skeptical, that is, when they are not much influenced by social pressure, states of anticoordination may arise. ${ }^{5}$ In these states, each of the two layer coordinates in a different strategy. On the other hand, when agents have herding behavior in response to social pressure, chimera-like dynamical states can emerge in an asymmetric coordination game. In such chimera states, there is coexistence of a homogeneous state of coordination in one layer with a heterogeneous state of coexistence of strategies in the other layer.

Based on the game-theoretical approach to coordination games, many theoretical, numerical, and experimental studies have been done to elucidate the factors that favor the emergence of coordination, ${ }^{2,7,8}$ such as the range of payoffs, the strategy updating rules, and the structures of the network. ${ }^{9-11}$ There is some experimental evidence of the effect of local interactions as found in Refs. 12-14, the effect of social influence as reputation on the efficiency results, ${ }^{15}$ and the effect of intra-group communication. ${ }^{16}$ In the context of dynamical networks, there are studies of network formation and social coordination. ${ }^{17,18}$ A simulation study of coordination games on dynamical networks finds states of polarization (formation of clustering of coexisting communities, each of them with a different strategy) in the symmetric coordination game. ${ }^{19}$ However, it is difficult to find studies that explore the origin and robustness of states of anticoordination between two fixed populations. Likewise, the literature about chimera states is vast in the field of coupled dynamical systems in networks of interaction, ${ }^{20-27}$ but few studies address the existence of chimera states in social networks. ${ }^{6,28,29}$ Anticoordination and chimera states are difficult to find in a single component network. However, the framework of a multilayer network seems to be the natural baseline for the emergence of these non-trivial states. Indeed, these states are observed in a fully connected two-layer network with particular settings of the initial distribution of strategies, the magnitude of social influence, and the parameters of the coordination game. ${ }^{6}$ The aim of this paper is to study the robustness of these states in settings beyond a fully connected network. We will consider random [Erdös-Rényi (ER)] networks inside and between layers. These networks are formed by connecting agents in a random manner. ${ }^{30}$ We will explore the effects of connectivity measured by the mean number of links per node (mean degree). In this way, we introduce local effects not considered in the fully connected network. We will show that there are threshold values of the mean degree so that below such threshold values, that is, when local effects are more important, anticoordination and chimera states no longer exist. We also discuss the different importance of local effects in the inter-layer and intra-layer connectivity. In addition, we also discuss the effects of locality in the problem of which of the two competing coordination states is selected in an asymmetric coordination game.

The paper is structured as follows. Section II describes the model and the asymptotic states. Section III shows the results and discusses the effects of local connectivity. Section IV summarizes our conclusions.

\section{MODEL}

We consider the two-layer network model proposed in Ref. 1 in which each individual or agent belongs to two different networks. The inter-layer network or the coordinating game playing network is one formed by the links between layers, and the intra-layer network or the social-based learning network is one formed by the links inside the layer. This implies that there is a single coordinating game playing network and two social-based learning networks; see Fig. 1.

For our numerical simulations, we will fix the system size $N=N_{A}+N_{B}=1000$, where $N_{A}$ and $N_{B}$ are the sizes of each layer. The network structures correspond to random (Erdös-Rényi) networks ${ }^{30}$ with the mean number of connections per node (mean degree) $\left\langle k_{A}\right\rangle$ and $\left\langle k_{B}\right\rangle$ inside layer $A$ and layer $\mathrm{B}$, respectively, and the mean degree $\left\langle k_{A B}\right\rangle$ for the inter-layer $E R$ random network.

In a fully connected network, the average number of links per agent in each layer is given by $\left\langle k_{A}\right\rangle=\left\langle k_{B}\right\rangle=(N / 2-1)$, and the number of links that connect layer $A$ and $B$ is given by $L_{A B}=(N / 2)^{2}$, with the average degree of inter-layer connections given by $\left\langle k_{A B}\right\rangle=N / 2$.

Each agent plays a two-person two-strategy coordination game with each player in the playing network using the same action for all those games. This coordination game can be of two types. It is symmetric when each agent receives a payoff of 1 for mutual coordination in strategy, namely, $L$ or $R$, and zero when they play opposite strategies. It is asymmetric when agents get a payoff of 1 by coordinating in $L$ and a payoff of 2 by coordinating in $R$. When they play opposite strategies, the one playing $L$ gets a payoff of 0 and the one playing $R$ gets a payoff of $-b$ for $b>0$; see Table I.

From a game-theoretical approach, the two states of coordination, in $L$ or $R$, are the two Nash equilibria in pure strategies of this game. This classic game illustrates the problem of equilibrium selection. The challenge for each agent in our model is to

\section{LAYER A LAYER B}

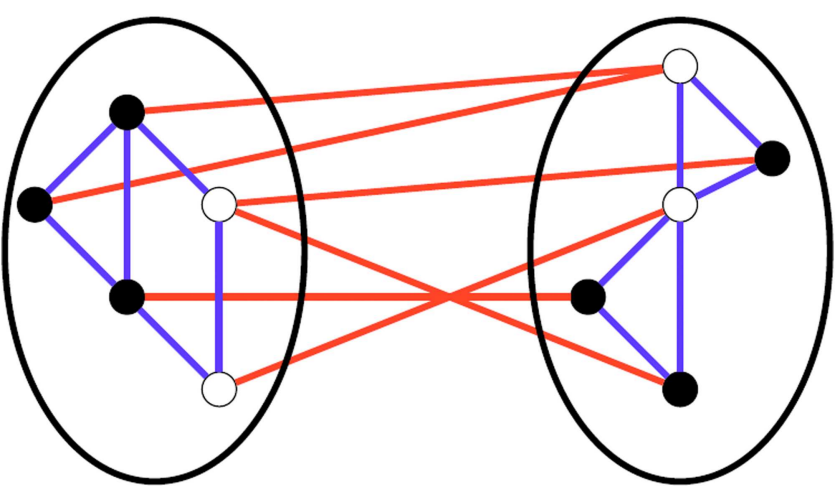

FIG. 1. An example of a two-layer network. The nodes are connected to each other in a pairwise manner both inside the layers and between the layers $A$ and B. Red lines describe the playing network (i.e., inter-layer edges), and the blue lines describe the two social-based networks (intra-layer edges). The black nodes describe the agents playing strategy $L$ and white nodes the agents playing strategy $\mathrm{R}$ in the coordination game. 
TABLE I. Payoff matrix for a two-person, two-strategy coordination game. For the symmetric coordination game, $s=0$ and $b=0$, and for the asymmetric coordination game, $s=1$ and $b>0$.

\begin{tabular}{|c|c|c|}
\hline & $\mathbf{L}$ & $\mathbf{R}$ \\
\hline $\mathbf{L}$ & 1,1 & $0,-b$ \\
\hline $\mathbf{R}$ & $-b, 0$ & $1+s, 1+s$ \\
\hline
\end{tabular}

coordinate either in $L$ or $R$ with all her neighbors in the playing network in the symmetric coordination game and to coordinate on $R$ (larger payoff) in the asymmetric game. Each player searches for receiving an aggregate payoff equal to the size of her neighborhood in the symmetric game or double the size of her neighborhood in the asymmetric game. In the asymmetric game, the strategy $R$ is called the Pareto-dominant strategy since the payoff by coordinating in $R$ is greater than the payoff by coordinating in $L$. By Harsanyi and Selten definition in Ref. 31, the strategy $R$ becomes risky when $b>1$, and, as a result, agents end playing the strategy $L$, called the risk-dominant strategy.

In the social-based learning network, agents observe the popularity of the strategy they currently play in the coordination game. This aspect can be described by defining a threshold $T$ as a gauge of the skepticism about the popularity of the opposite strategy, $T \in[0,1]$. According to the threshold $T$, the population is called herding when $T<0.5$. In this case, agents are easily influenced by the popularity of the opposite strategy playing by their partners. Also, it is called skeptical when $T>0.5$. These agents readily reject the social pressure exerted by the players with an opposite strategy. The social interaction works as follows: when a player $i$ observes the proportion of her neighbors, $d_{i}$, who are playing the opposite strategy to hers in her social-based network, she measures how popular her strategy is comparing $d_{i}$ with $T$. For instance, if $d_{i}>T$, the player $i$ feels socially unsatisfied about the strategy she is currently playing.

At each time step, the timing of the model can be described in three parts. First, each agent plays a coordination game in a pairwise manner with each neighbor in her playing network and gets an aggregate payoff. Second, she looks at the popularity of her strategy comparing $d_{i}$ with $T$. Third, she decides whether or not to revise her strategy.

The criterion to change the strategy is based upon two basic rules: how well her performance is in terms of the payoff obtained in her playing network and how well her current strategy is accepted in her social-based network. Accordingly, there are four feasible results that depend upon the aggregate payoff of the agent $i$ denoted by $\pi_{i}$ and the relation between $d_{i}$ and $T$.

The equality $\pi_{i}=(1+s) k_{i}$ means that the player $i$ coordinates with all her neighbors in the playing network where $k_{i}$ (degree of node $i$ in the inter-layer network) is the size of her neighborhood in the playing network. When $s=0$ (symmetric coordination game), the coordination is either in $L$ or $R$, and the coordination is only in the strategy $R$ when $s=1$ (asymmetric coordination game). The inequality $d_{i}<T$ means that the proportion of neighbors in her social-based network who play the opposite strategy as she does is low enough so that the player $i$ feels confident with the popularity of her current strategy. Then, the three possible situations for agent $i$ are as follows:

Pleased: when she is both socially $\left(d_{i}<T\right)$ and strategically $\left[\pi_{i}=(1+s) k_{i}\right]$ satisfied,

Partially pleased: when she is either socially $\left(d_{i}<T\right)$ or strategically $\left[\pi_{i}=(1+s) k_{i}\right]$ satisfied, and

Displeased: when she is both socially $\left(d_{i}>T\right)$ and strategically $\left[\pi_{i}<(1+s) k_{i}\right]$ unsatisfied.

\section{A. The strategy update rule}

The synchronous update rule in which each player can change her current strategy depends on the result of her social and strategic interactions. If after her strategic and social interactions, the agent is pleased, she remains with her current strategy. If the agent is partially pleased, she imitates the strategy of her best performing neighbor in her social-based intra-layer network when she has received a larger payoff than the player herself; otherwise, she remains with the same strategy. If the agent is displeased, she switches her current strategy to the opposite one.

This update rule captures the individual behavior in complex real-life situations taking into account both social and strategic environments. Once she takes a decision about her strategy according to the update rule described above, the process is repeated setting payoffs to zero.

\section{RESULTS AND DISCUSSION}

The analytical solutions of the mean-field equations in Ref. 6 describe the collective behavior of this evolutionary model. Depending upon the initial conditions and values of the parameters in a fully connected network, the system reaches one four possible different asymptotic states:

1. States of coordination: $\{(L, L),(R, R)\}$. The first component of $(L, L)$ corresponds to the asymptotic state in layer A in which agents end playing the strategy $L$ and the second component corresponds to the asymptotic state in layer B in which agents end playing the strategy $L$. Similarly, the components of $(R, R)$ correspond to the asymptotic states in which agents end playing the strategy $R$ in layer $\mathrm{A}$ and layer $\mathrm{B}$, respectively.

2. States of anticoordination: $\{(L, R),(R, L)\}$. The asymptotic state $(L, R)$ corresponds to a coordination state in layer $\mathrm{A}$ in the strategy $L$ and a coordination state in layer B in the strategy $R$. In the same way, the asymptotic state $(R, L)$ corresponds to a coordination state in layer $A$ in the strategy $R$ and a coordination state in layer B in the strategy $L$.

3. A family of the dynamical state of coexistence of strategies with temporal oscillations: $\left\{\left(u_{a}, v_{b}\right)\right\}$ where $u_{a}=\{u, 1-u\}$ represents a dynamical state with an oscillation from $u$ to $1-u$ of the proportion of agents playing $R$ in layer $\mathrm{A}$ and $v_{b}=\{v, 1-v\}$ 
represents a dynamical state with an oscillation from $v$ to $1-v$ of the proportion of agents playing $R$ in layer $\mathrm{B}$, for $u, v \in(0,1)$.

4. A family of chimera states $\left\{\left(u_{a}, L\right),\left(L, v_{b}\right)\right\}$ where $u_{a}=\{u, 1-u\}$ and $v_{b}=\{v, 1-v\}$ represent dynamical states with oscillations of the proportion of agents playing $R$ in layer $\mathrm{A}$ and layer $\mathrm{B}$, respectively, for $u, v \in(0,1)$ and $L$ represents the state of coordination reached by the other layer.

The asymptotic anticoordination and chimera states are two highlights of this two-layer network. These states do not occur in a single isolated network. The analytical results in Ref. 6 show that the chimera states appear only in herding populations, i.e., $T<0.5$, when the coordination game is asymmetric, while the states of anticoordination appear only in skeptical populations, i.e., $T>0.5$ for either a symmetric or an asymmetric coordination game. The asymptotic state and the size of the basin of attraction of these states are determined by the particular initial conditions of strategies, the parameter $T$, and the parameters $s$ and $b$ of the coordination game.

We introduce in Ref. 1 an order parameter $n_{A B}$ giving the proportion of inter-layer active links, i.e., links among agents playing opposite strategies in different layers.

When the order parameter $n_{A B}=0$, the system reaches a coordination state where the agents in both layers play the same strategy. This corresponds to $(L, L)$ and $(R, R)$.

For $0<n_{A B}<1$, the system reaches a dynamical state of coexistence of strategies with oscillations corresponding to $\left\{\left(u_{a}, v_{b}\right)\right\}$ as described above.

For the chimera states, the order parameter $n_{A B}$ displays a dynamical behavior with the oscillation $u_{a}$ or $v_{b}$ corresponding to $\left\{\left(u_{a}, L\right),\left(L, v_{b}\right)\right\}$, respectively, since one of the layers reaches a coordination state in $L$ and the other layer reaches a dynamical state of coexistence of strategies with oscillations described as $u_{a}$ or $v_{b}$. The order parameter $n_{A B}=1$ corresponds to a situation in which the agents in each layer play opposite strategies. The asymptotic states are anticoordination states $(L, R)$ and $(R, L)$.

A complete analysis of these asymptotic states when the network is fully connected can be found in Ref. 6. Our aim in this paper is to focus on the effects of locality occurring in non-fully connected networks of interaction. Local effects will be shown to prevent the existence of asymptotic states of anticoordination, chimera states, and states of coordination $(L, L)$ when such outcome becomes riskdominant in the asymmetric coordination game. The notation used for the proportion of agents playing the strategy $R$ in layer $A$ is $x_{a a}$ and $x_{b b}$ in layer $B$.

\section{A. Symmetric coordination game: Local connectivity destroys asymptotic states of anticoordination}

In this section, we analyze the effect of local connectivity in the symmetric coordination game, $s=b=0$. We study the cases when the mean degrees inside and between layers are the same, i.e., $\langle k\rangle=\left\langle k_{A}\right\rangle=\left\langle k_{B}\right\rangle=\left\langle k_{A B}\right\rangle$ and when they are different, i.e., $\left\langle k_{A}\right\rangle=\left\langle k_{B}\right\rangle \neq\left\langle k_{A B}\right\rangle$.

For an easier analysis, we introduce a normalized $\left\langle K_{j}\right\rangle=\frac{\left\langle k_{j}\right\rangle}{k_{\max }}$, where $k_{\text {max }, j}$ is the maximum number of neighbors per agent in layer $A$ or $B$ for $j=A, B$ or between layers for $j=A B$. Specifically, $k_{\text {max }, j}=\left(\frac{N}{2}-1\right)$ for $j=A, B$, and $k_{\text {max }, j}=\frac{N}{2}$ for $j=A B$; as stated

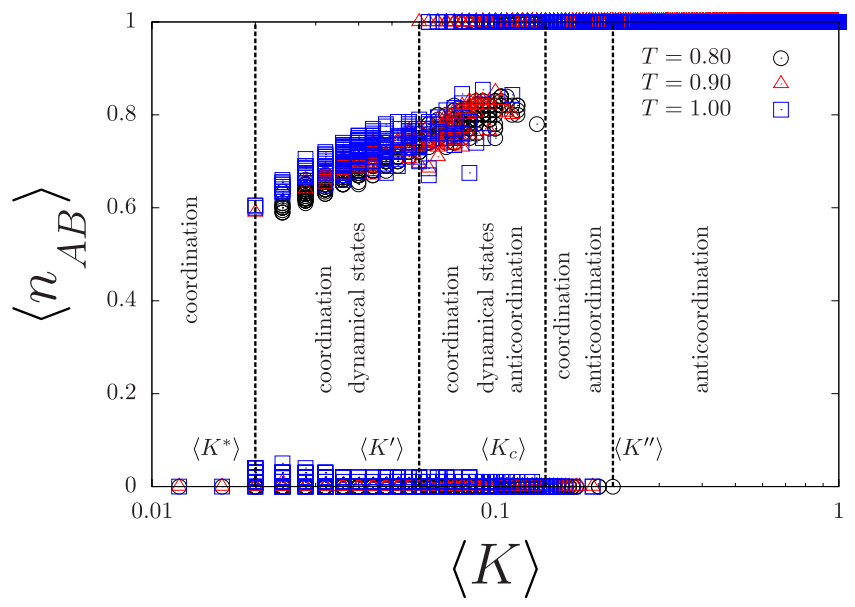

FIG. 2. Bifurcation diagram of $\left\langle n_{A B}\right\rangle$ as a function of a normalized $\langle K\rangle=\left\langle K_{A}\right\rangle$ $=\left\langle K_{B}\right\rangle=\left\langle K_{A B}\right\rangle$. The initial condition corresponds to $x_{a a}=0.6$ and $x_{b b}=0.4$ for different values of $T$. Dashed lines correspond to the thresholds of $\langle K\rangle$ for the different final configurations of the system.

above, $N=N_{A}+N_{B}$. Then, we have that $\left\langle K_{j}\right\rangle=\frac{2\left\langle k_{j}\right\rangle}{N-2}$ for $j=A, B$ and $\left\langle K_{j}\right\rangle=\frac{2\left\langle k_{j}\right\rangle}{N}$ for $j=A B$. When the mean degree does not differ inside and between layers, we set $\langle K\rangle=\left\langle K_{A}\right\rangle=\left\langle K_{B}\right\rangle=\left\langle K_{A B}\right\rangle$. To determine the value of $\langle K\rangle$ for which the anticoordination absorbing state emerges, we have plotted in Fig. 2 the bifurcation diagram of the mean value of $n_{A B}$ over 500 independent realizations of the initial conditions. It is shown as a function of $\langle K\rangle$ for different values of $T$. The distribution of the initial condition is such that in a fully connected network, the system goes to a state of anticoordination. Figure 2 displays the existence of a threshold value $\left\langle K^{\prime \prime}\right\rangle$, beyond which $n_{A B}=1$, indicating that the system only reaches an anticoordination state as it happens in the fully connected network. Furthermore, numerical simulations also show that in the range between $\left\langle K^{*}\right\rangle$ and $\left\langle K^{\prime \prime}\right\rangle$, different asymptotic states take place in the system with domains of bistability or tristability involving anticoordination, coordination, and dynamical states. For $\langle K\rangle\left\langle\left\langle K^{*}\right\rangle\right.$, the system only reaches states of coordination. These results show the effect of locality that prevents anticoordination and favors coordination as the number of links in the networks decreases.

To elucidate what is happening in the system for different values of $\langle K\rangle$, we have plotted in Fig. 3 the frequency histogram of the value of the order parameter $n_{A B}$ sampled by 500 realizations for $T=0.85$. It illustrates the different behaviors that are taking place on the system: The system goes to a state of coordination for $\langle K\rangle=0.02$, to a state of anticoordination for $\langle K\rangle=0.30$, and in the range $\langle K\rangle \in(0.02,0.30)$, the system reaches different configurations with possible different asymptotic coordination, anticoordination, or dynamical states.

An interesting question is about the probability that the system reaches an anticoordination state for a given value of $\langle K\rangle$ and a fixed $T$. Figure 4 shows such probability for different values of $T$ obtained from numerical simulations. This probability increases as $T$ increases for a given value of $\langle K\rangle$. Also, we can observe that the 

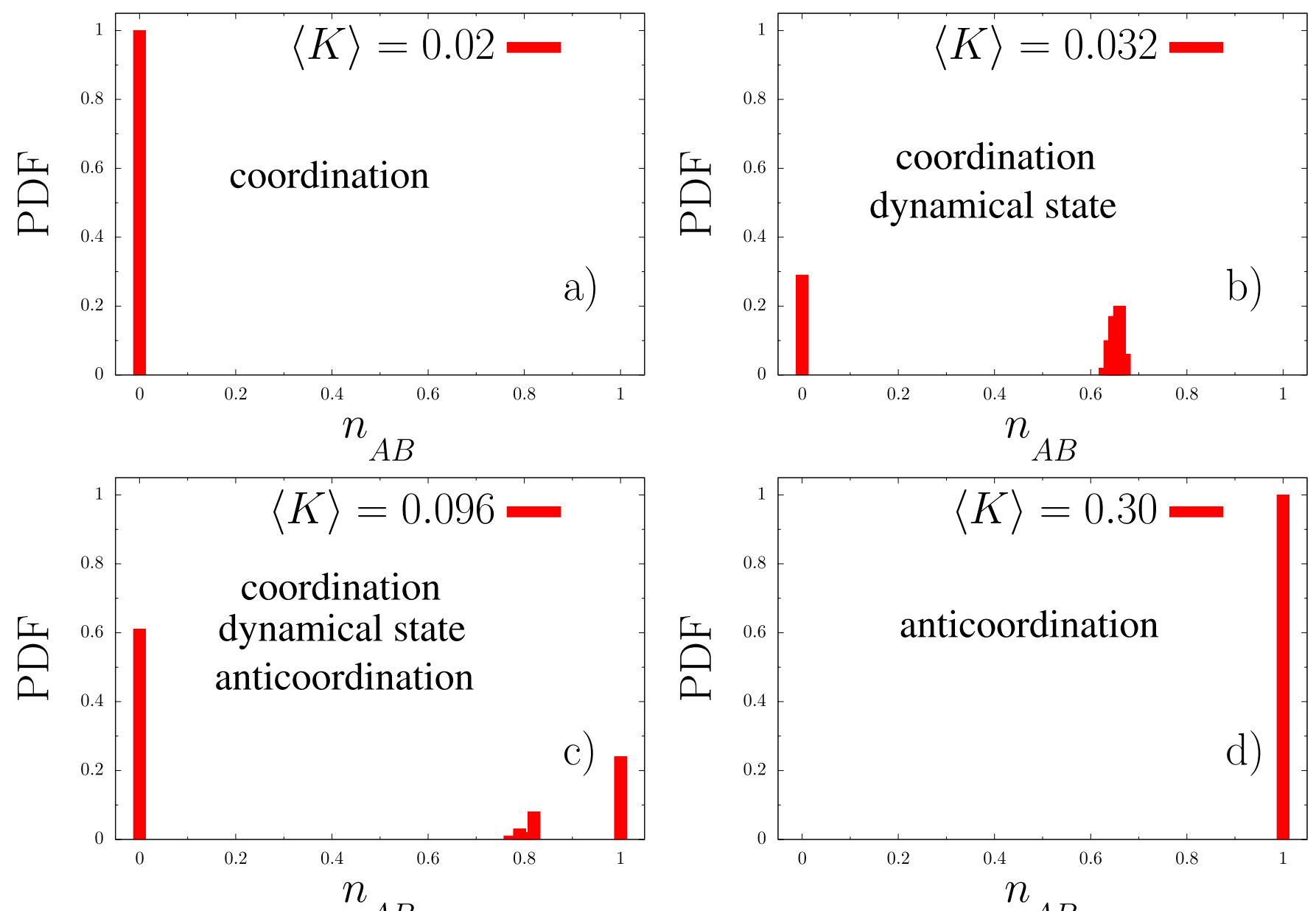

$A B$

FIG. 3. Frequency histogram of $n_{A B}$ for different values of $\langle K\rangle=\left\langle K_{A}\right\rangle=\left\langle K_{B}\right\rangle=\left\langle K_{A B}\right\rangle$, where $T=0.85$ and for an initial distribution of strategies, $x_{a a}=0.6$ and $x_{b b}=0.4$, which in a fully connected network, the system goes to an anticoordination state. The values are $\langle K\rangle=0.02$ in (a), $\langle K\rangle=0.032$ in (b), $\langle K\rangle=0.096$ in (c), and $\langle K\rangle=0.30$ in (d). The histogram is built on a set of 500 independent realizations of the initial conditions.

threshold value of $\langle K\rangle$ for which the system has zero probability to reach an anticoordination state increases as $T$ decreases. There is a threshold value of $\langle K\rangle$ below which the system never reaches an anticoordination state for any value of $T$. Again, this shows that locality prevents the existence of asymptotic anticoordination states. We can also determine the threshold value $\left\langle K^{\prime}\right\rangle$ above which there are possible anticoordination states. This is shown in Fig. 5 as a function of the threshold value $T$, indicating that skepticism favors anticoordination states: As $T$ increases, the threshold value $\left\langle K^{\prime}\right\rangle$ decreases monotonically.

So far, we have considered values of the mean degree inside and between layers alike; i.e., $\langle k\rangle=\left\langle k_{A}\right\rangle=\left\langle k_{B}\right\rangle=\left\langle k_{A B}\right\rangle$. We now consider what happens when this requirement is relaxed, and the mean degree of the intra-layer network is different from the mean degree of the inter-layer network. Therefore, we analyze the relative importance of the local effects in the learning process (intra-layer) and in playing the coordination game (inter-layer).

Figure 6 shows numerical results for $T=0.85$. It shows the probability that the system reaches a final state of anticoordination as a function of the intra-layer normalized mean degree $\langle K\rangle=\left\langle K_{A}\right\rangle=\left\langle K_{B}\right\rangle$ for different values of the inter-layer normalized mean degree $\left\langle K_{A B}\right\rangle$. It appears that this probability becomes larger as the inter-layer mean degree increases for any value of $\langle K\rangle$. Besides, Fig. 7 shows, in the plane $\langle K\rangle,\left\langle K_{A B}\right\rangle$, a threshold line from a domain of zero probability of anticoordinating to a domain in which anticoordination is possible. The transition to possible anticoordination is largely independent of $\langle K\rangle=\left\langle K_{A}\right\rangle=\left\langle K_{B}\right\rangle$ except for very small values of $\left\langle K_{A B}\right\rangle$. This indicates that although little connectivity in the whole system favors coordination and much connectivity favors anticoordination, local effects in the intra-layer (learning) 


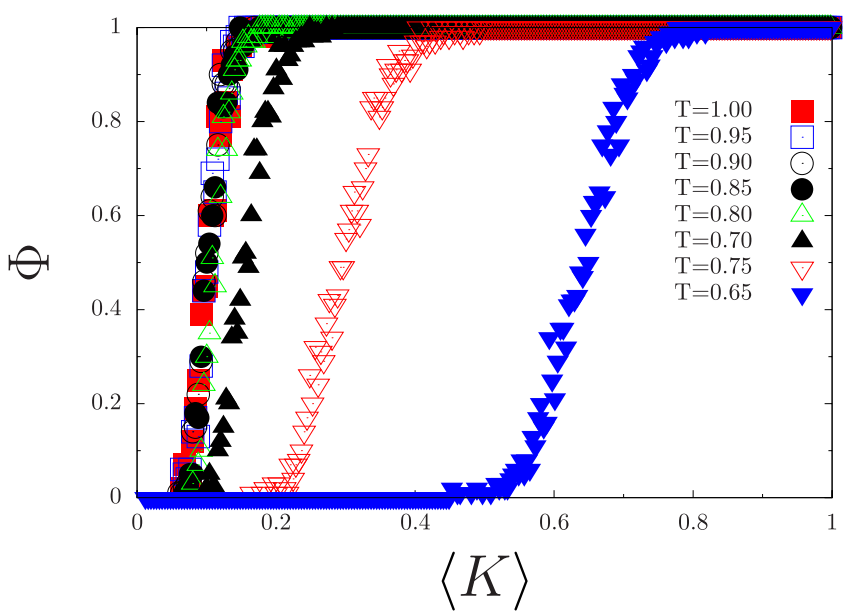

FIG. 4. Probability that the system reaches a final state of anticoordination as a function of $\langle K\rangle$ for different values of $T$.

network are more important than those in the inter-layer (coordination game) network to destroy the possibility of anticoordination states.

\section{B. Asymmetric coordination game: Local connectivity destroys asymptotic chimera states}

The effects of local connectivity in chimera states turn out to be similar to what we have found for anticoordination states. When the normalized mean degree inside and between layers are the same, i.e., $\langle K\rangle=\left\langle K_{A}\right\rangle=\left\langle K_{B}\right\rangle=\left\langle K_{A B}\right\rangle$, there exists a minimum and a maximum threshold value of $\langle K\rangle$ such that below the minimum value, the system does not reach a chimera state, and above the maximum value, the system reaches only chimera states. As mentioned above, the chimera states are observed only in herding populations, i.e., $T<0.5$, and when the coordination game is asymmetric. Figure 8 shows the probability that the system reaches chimera states, $\Phi_{c h}$, as

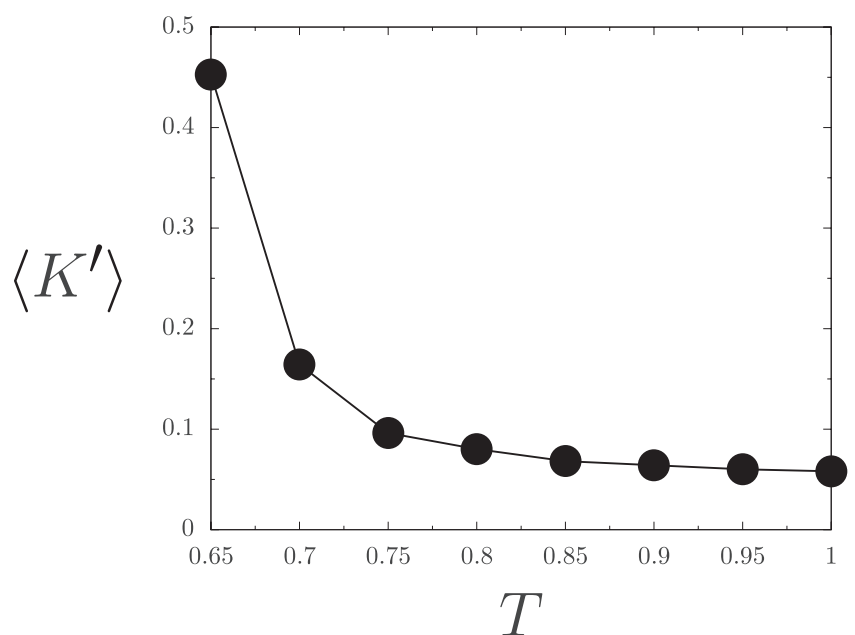

FIG. 5. The critical value $\left\langle K^{\prime}\right\rangle$ in which the system can end in a state of anticoordination as a function of $T$.

a function of a normalized $\langle K\rangle$ for parameter values for which, in a fully connected network, the system goes to a chimera state. Simulation results show that the only asymptotic states reached by the system when it does not reach a chimera state are dynamical states of coexistence of strategies.

Results when the normalized mean degree is different for the intra- and inter-layer networks are shown in Fig. 9. Figure 9(a) shows the probability that the system reaches a chimera state as a function of a normalized mean degree $\left\langle K_{A B}\right\rangle$ for different values of normalized mean degree $\left\langle K_{A}\right\rangle=\left\langle K_{B}\right\rangle$. The probability continuously increases as $\left\langle K_{A B}\right\rangle$ increases for relatively high intra-layer mean degrees. However, for a low intra-layer mean degree, the probability of reaching a chimera state becomes zero for any value of $\left\langle K_{A B}\right\rangle$. Besides, Fig. 9(b) shows the probability that the system reaches a chimera state as a function of a normalized $\left\langle K_{A}\right\rangle=\left\langle K_{B}\right\rangle$ for different values of $\left\langle K_{A B}\right\rangle$. In this case, this probability has a discontinuous

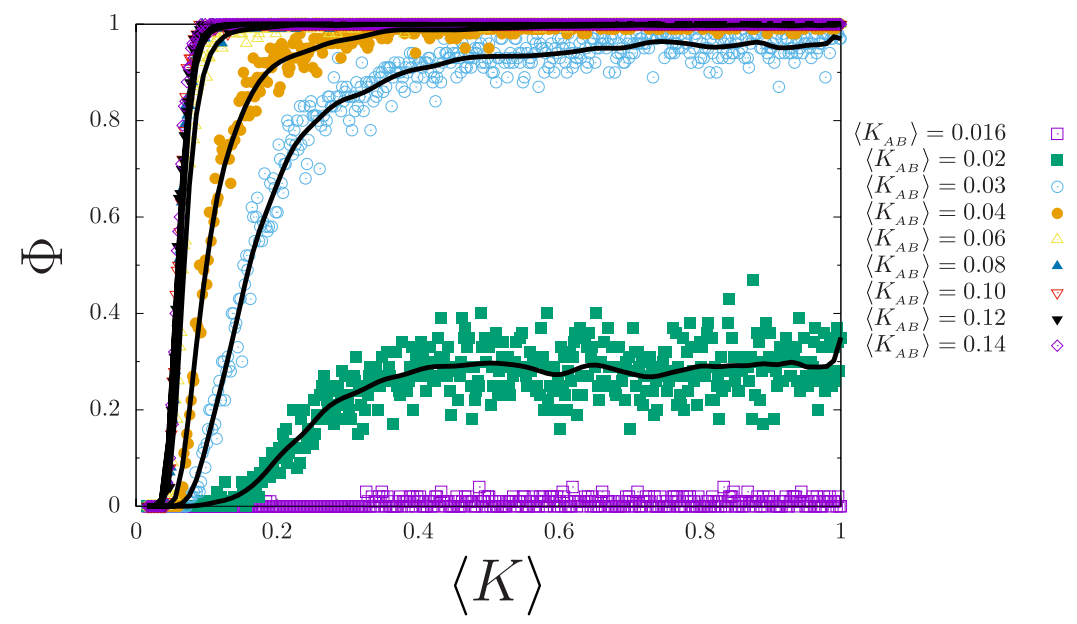

FIG. 6. Probability that the system reaches a final state of anticoordination as a function of $\langle K\rangle=\left\langle K_{A}\right\rangle=\left\langle K_{B}\right\rangle$ for different values of $\left\langle K_{A B}\right\rangle$. 


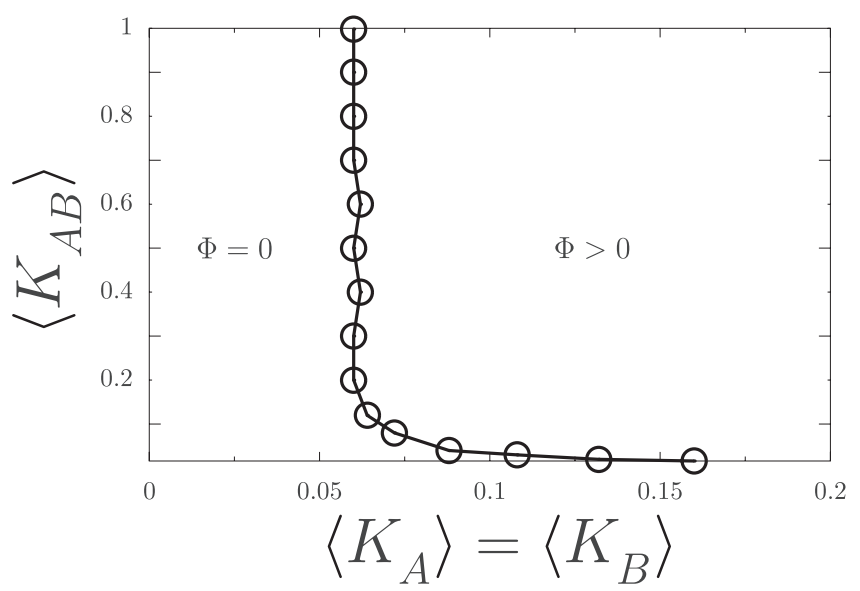

FIG. 7. Probability that the system reaches a final state of anticoordination in the plane $\left\langle K_{A B}\right\rangle,\langle K\rangle$ for $N=N_{A}+N_{B}=1000$. The left side of the line, $\Phi=0$, and the right side of the line, $\Phi>0$.

jump for relatively high inter-layer mean degrees. However, the probability is zero for all values of $\left\langle K_{A}\right\rangle=\left\langle K_{B}\right\rangle$ when the interlayer mean degree is low. Therefore, local effects are important in both networks, the intra-layer (learning) network as well as in the inter-layer (coordination game) network.

However, the effect shown in Fig. 8 is more associated with the inter-layer coordination locality, Fig. 9(a), than with the intra-layer learning locality, Fig. 9(b). The discontinuity jump shows the slight effect associated with the intra-layer locality on the prevention of chimera states.

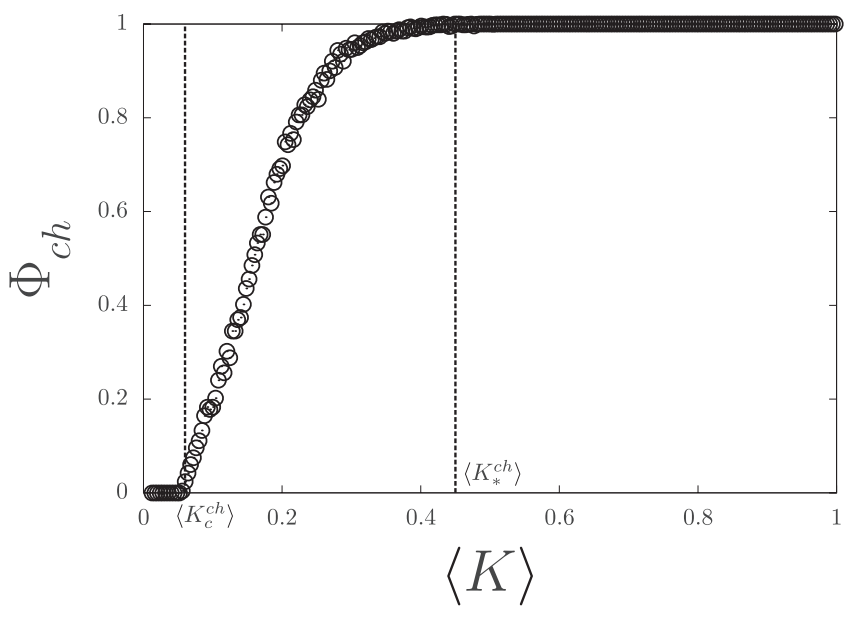

FIG. 8. Probability of chimera states vs a normalized $K$ for $T=0.25$ with initial conditions $x_{a a}=0.9, x_{b b}=0.4$, and $b=3$.
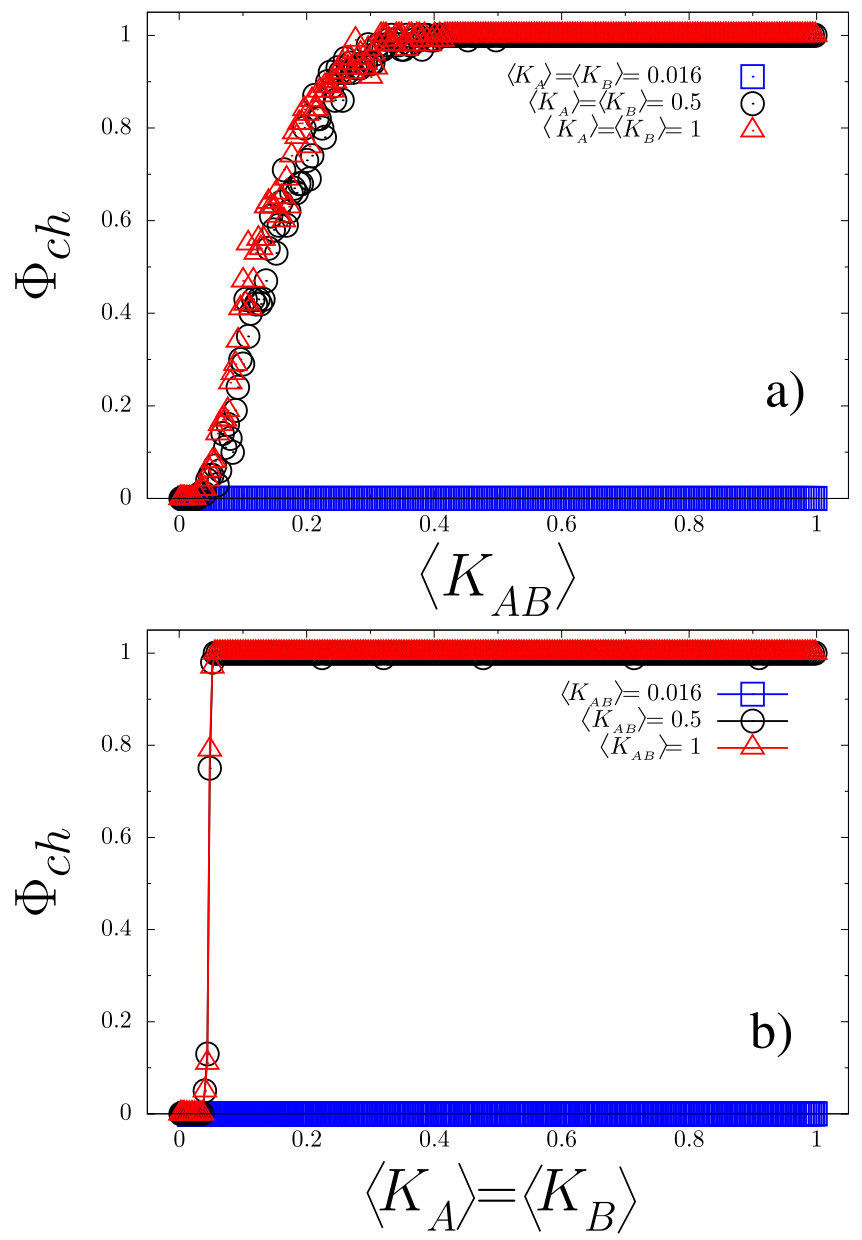

FIG. 9. Panel (a): Probability that the system reaches a chimera state as a function of a normalized $\left\langle K_{A B}\right\rangle$ for $\left\langle K_{A}\right\rangle=\left\langle K_{B}\right\rangle=0.016,0.5$, and 1. Panel (b): Probability that the system reaches a chimera state as a function of a normalized $\left\langle K_{A}\right\rangle=\left\langle K_{B}\right\rangle$ for $\left\langle K_{A B}\right\rangle=0.016,0.5$, and 1 . Here, $T=0.25$ with initial conditions $x_{a a}=0.9, x_{b b}=0.4$, and $b=3$.

\section{Asymmetric coordination game: Local connectivity and coordination-Pareto dominance vs risk dominance}

In the game-theoretical model of a two-person two-strategy coordination game, the equilibrium selection principles postulated by Harsanyi and Selten ${ }^{31}$ establish that coordination occurs in the Pareto-dominant strategy $R$ when $b<1$, while coordination occurs in the risk-dominant strategy $L$ for $b>1$. This is the result that we found in our model of a fully connected two-layer network with strategical and social interactions for the asymmetric coordination game. What we show here is how local connectivity favors the Pareto-dominant strategy when in the fully connected two-layer network, the risk-dominant strategy is the asymptotic outcome. 


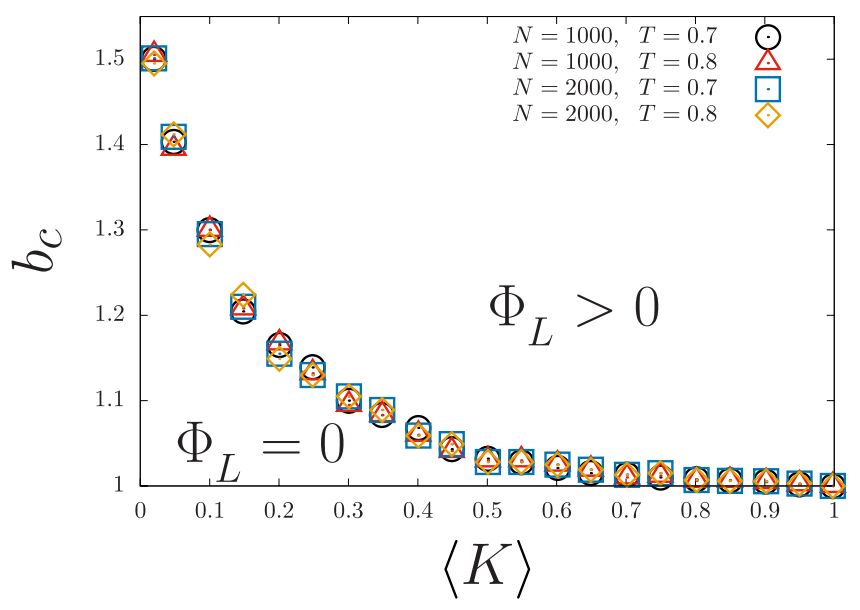

FIG. 10. Threshold of the parameter $b$ that makes the strategy $L$ the risk-dominant strategy as a function of a normalized mean degree $\langle K\rangle=\left\langle K_{A}\right\rangle=\left\langle K_{B}\right\rangle$ $=\left\langle K_{A B}\right\rangle$ for different sizes of $N$ and $T$ with initial conditions $x_{a a}=x_{b b}=0.5$.

In a fully connected two-layer network, by choosing symmetric initial conditions $\left(x_{a a}=x_{b b}=0.5\right)$, the system reaches a dynamical state of coexistence of strategies when $T<0.5$ and a state of full coordination on the risk-dominant strategy $L$ when $T>0.5$ and $b>1$. Focusing on this latter case, we analyze the effects of local connectivity. When the intra-layer and inter-layer mean degrees are equal, Fig. 10 shows the threshold value of the parameter $b$ such that for $b>b_{c}$, there is a positive probability, $\Phi_{L}>0$, to reach an asymptotic state of coordination in the risk-dominant strategy $L$. Otherwise, for $b<b_{c}$, there is zero probability of reaching this state: $\Phi_{L}=0$. It shows that the threshold $b_{c}$ increases as the normalized $\langle K\rangle$ decreases regardless of the values of $N$ and $T$. Locality implies that a larger $b$ is needed for coordination in the risk-dominant strategy. Figure 11 shows the probability of reaching a state of coordination on $L$ as a function of $b$ when the intra-layer and interlayer mean degrees are different. Three cases are shown: (1) fully connected networks, (2) fully connected intra-layer networks, and

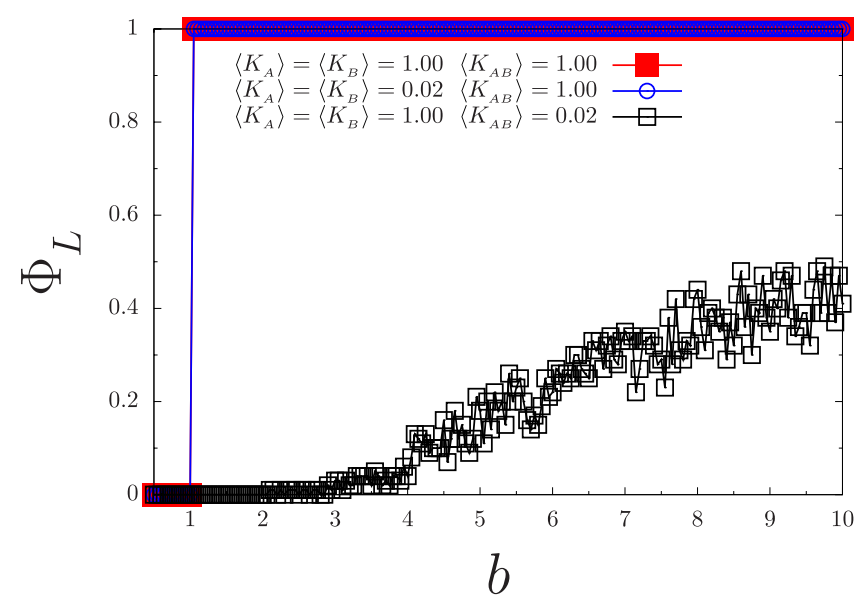

FIG. 11. Probability that the system reaches a coordination state in $L$ as a function of $b$ for three cases: (1) fully connected network, $\left\langle K_{A}\right\rangle=\left\langle K_{B}\right\rangle=\left\langle K_{A B}\right\rangle$ $=1$ (red squares); (2) fully connected inter-layer network, $\left\langle K_{A B}\right\rangle=1$ and $\left\langle K_{A}\right\rangle$ $=\left\langle K_{B}\right\rangle=0.02$ (blue circles); and (3) fully connected intra-layer networks, $\left\langle K_{A}\right\rangle=\left\langle K_{B}\right\rangle=1$ and $\left\langle K_{A B}\right\rangle=0.02$ (black squares). The initial conditions are $x_{a a}=x_{b b}=0.5$ and $T=0.8$.

(3) fully connected inter-layer network. We note that the important local effects are those of the inter-layer network, while local effects in the intra-layer network do not modify the threshold value $b_{c}=1$ of the fully connected network. This result is qualitatively different from what we found for anticoordination states where the most important local effects are those associated with the intra-layer learning network. However, the local effects are quite similar to what we observe in chimera states where even though they are important for both networks, the inter-layer local effects seem more important in avoiding chimera states than the intra-layer local effects.

A more detailed description of local effects associated with the inter-layer network is given in Fig. 12 that shows the probability that the system reaches a coordination state in $L$ for different values of a normalized $\left\langle K_{A B}\right\rangle$ and a fully connected intra-layer network; i.e., $\left\langle K_{A}\right\rangle=\left\langle K_{B}\right\rangle=1$. In summary, the inter-layer local

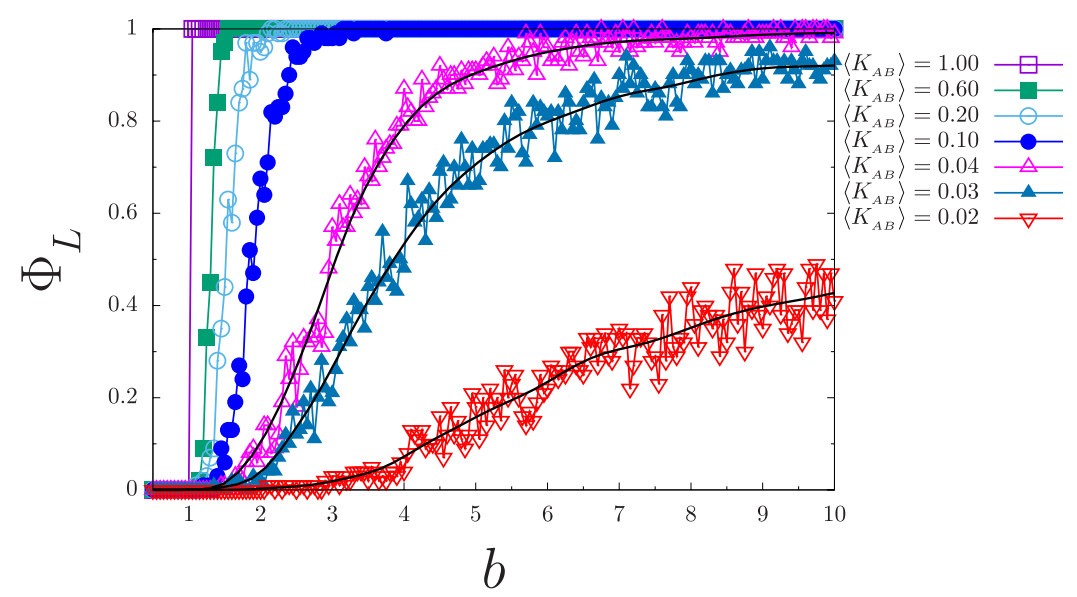

FIG. 12. Probability that the system reaches a coordination state in $L$ as a function of $b$ for different inter-layer mean degree, $\left\langle K_{A B}\right\rangle$, when the intra-layer networks are fully connected, $\left\langle K_{A}\right\rangle=\left\langle K_{B}\right\rangle=1$. The initial conditions are $x_{a a}=x_{b b}=0.5$ and $T=0.8$. 
connectivity reduces the probability of reaching coordination in the risk-dominant strategy $L$ and in turn rises the probability of reaching coordination on the Pareto-dominant strategy.

\section{CONCLUSIONS}

For a two-layer model of learning and coordination dynamics and for the case of a population of skeptical individuals in fully connected networks, the system may reach an asymptotic state of anticoordination when agents play a symmetric or an asymmetric coordination game between layers. On the other hand, for a herding population, the system may reach an asymptotic chimera state when agents play an asymmetric coordination game. These two asymptotic states of anticoordination and chimera states are not observed in a single component network. Therefore, the setting of a two-layer structure favors the emergence of these asymptotic states. Nevertheless, this setting is not a sufficient condition, and we have discussed their robustness when including local effects with random networks that bring in the effect of local connectivity.

We have shown that important local effects associated with low connectivity destroy anticoordination states for initial distributions of strategies such that, in a fully connected network, this leads the system to a state of anticoordination. We find threshold values of the mean degree below which the system may reach other states such as coexistence of strategies and coordination. We also find a threshold value of the mean degree, below which the only asymptotic state reached is coordination. Thus, local connectivity not only prevents final configurations of anticoordination but also favors coordination. The transition from anticoordination to coordination when increasing local effects is through intermediate phases with bistability and tristability among coordination, anticoordination, and dynamical states. We have also found that local effects in the intra-layer learning network are more important to destroy anticoordination states than local effects in the inter-layer network. This means that for any mean degree of the inter-layer network, the probability of reaching an asymptotic state of anticoordination decreases as the mean degree of the intra-layer network decreases. Less connectivity for the social learning process prevents states of anticoordination.

Similar results are observed for the case of chimera states, with local connectivity inside and between layers preventing the existence of chimera states. The probability of reaching an asymptotic chimera state decreases as local connectivity decreases. In the case in which intra-layer and inter-layer mean degrees are different, less social connectivity (lower mean degree of the intra-layer network) prevents the existence of chimera, but less strategic connectivity (lower mean degree of the inter-layer network) also prevents the existence of asymptotic chimera states. This means that when the intra-layer mean degree is high, the inter-layer mean degree must be low to prevent existence of chimera states and conversely. Still, the local effect that comes from the inter-layer coordination network is more important in reducing chimera states than the local effect of intra-layer connectivity.

We have also considered local effects in the problem of the selection of coordination equilibria. When agents play the asymmetric coordination game in a fully connected network, the riskdominant equilibrium ( $L$ coordination) is selected for $b>1$, similar to the game-theoretical approach. Local connectivity prevents this inefficient outcome, and the Pareto-dominant equilibrium ( $R$ coordination) can still be selected for $b>1$. The threshold value of $b$ for which the strategy $L$ becomes risk-dominant increases as connectivity decreases. For this issue, the most important local effect comes from the inter-layer connectivity: Less connectivity associated with network in which the coordination game is played prevents selection of the risk-dominant equilibrium in favor of the Pareto dominance.

\section{ACKNOWLEDGMENTS}

Haydée Lugo acknowledges financial support from Ministerio de Ciencia e Innovación (Spain) under Project No. PID2019109157RB-I00. Maxi San Miguel acknowledges financial support from Agencia Estatal de Investigación (AEI, MCIU, Spain) and Fondo Europeo de Desarrollo Regional (FEDER, UE) under Project PACSS (No. RTI2018-093732-B-C21/C22) and the Maria de Maeztu Program for units of Excellence in R\&D (No. MDM2017-0711)

\section{DATA AVAILABILITY}

The data that support the findings of this study are available from the corresponding author upon reasonable request.

\section{REFERENCES}

${ }^{1}$ H. Lugo and M. San Miguel, "Learning and coordinating in a multilayer network,” Sci. Rep. 5, 7776 (2015).

${ }^{2}$ S. Weidenholzer, "Coordination games and local interactions: A survey of the game theoretic literature,” Games 1(4), 551-585 (2010).

${ }^{3}$ N. E. Friedkin and E. C. Johnsen, Social Influence Network Theory (Cambridge University Press, New York, 2011).

${ }^{4}$ A. Flache, M. Mas, T. Feliciani, E. Chattoe-Brown, G. Deffuant, S. Huet, and J. Lorenz, "Models of social influence: Towards the next frontiers," J. Artif. Soc. Soc. Simul. 20(4), 2 (2017).

${ }^{5}$ J. C. González-Avella, H. Lugo, and M. San Miguel, "Coordination in a skeptical two-group population,” J. Econ. Interact. Coord. 14(1), 203-214 (2019).

${ }^{6}$ H. Lugo, J. C. González-Avella, and M. San Miguel, "Chimera and anticoordination states in learning dynamics," Front. Appl. Math. Stat. 5, 16 (2019).

${ }^{7}$ F. Vega-Redondo, Economics and the Theory of Games (Cambridge University Press, Cambridge, UK, 2003).

${ }^{8} \mathrm{~J}$. K. Goeree and C. A. Holt, "Coordination games," in The Encyclopedia of Cognitive Science (Nature Publishing Group, Macmillan, 2002), Vol. 2, pp. 204-208.

${ }^{9} \mathrm{~V}$. Buskens and C. Snijders, "Effects of network characteristics on reaching the payoff-dominant equilibrium in coordination games: A simulation study," Dyn. Games Appl. 6, 477-494 (2016).

${ }^{10}$ A. Antonioni, M. P. Cacault, R. Lalive, and M. Tomassini, "Coordination on networks: Does topology matter?,” PLoS One 8(2), e55033 (2013).

${ }^{11} \mathrm{M}$. Tomassini and E. Pestelacci, "Evolution of coordination in social networks: A numerical study,” Int. J. Mod. Phys. C 21, 1277-1296 (2010).

${ }^{12}$ A. Cassar, "Coordination and cooperation in local, random and small world networks: Experimental evidence,” Games Econ. Behav. 58, 209-230 (2007).

${ }^{13}$ C. Keser, K. M. Erhart, and S. Berninghaus, "Coordination and local interaction: Experimental evidence,” Econ. Lett. 59, 269-275 (1998).

${ }^{14} \mathrm{G}$. Ellison, "Learning, local interaction, and coordination," Econometrica 61, 1047-1071 (1993).

${ }^{15} \mathrm{~W}$. Raub and J. Weesie, "Reputation and efficiency in social interactions: An example of network effects," Am. J. Soc. 96, 626-654 (1990). 
${ }^{16} \mathrm{~J}$. V. Elten and S. P. Penczynski, "Coordination games with asymmetric payoffs: An experimental study with intra-group communication," J. Econ. Behav. Organ. 169, 158-188 (2020).

${ }^{17}$ S. Goyal and F. Vega-Redondo, "Network formation and social coordination," Games Econ. Behav. 2, 178-207 (2005).

${ }^{18} \mathrm{M}$. O. Jackson and A. Watts, "On the formation of interaction networks in social coordination,” Games Econ. Behav. 41, 265-291 (2002).

${ }^{19} \mathrm{M}$. Tomassini and E. Pestelacci, "Coordination games on dynamical networks," Games 1, 242-261 (2010)

${ }^{20} \mathrm{Y}$. Kuramoto and D. Battogtokh, "Coexistence of coherence and incoherence in nonlocally coupled phase oscillators," Nonlinear Phenom. Complex Syst. 5(4), 380-385 (2002).

${ }^{21}$ D. M. Abrams and S. H. Strogatz, "Chimera states for coupled oscillators," Phys. Rev. Lett. 93, 174102 (2004).

${ }^{22} \mathrm{H}$. Sakaguchi, "Instability of synchronized motion in nonlocally coupled neural oscillators," Phys. Rev. E 73, 031907 (2006).

${ }^{23} \mathrm{Y}$. Suda and K. Okuda, "Persistent chimera states in nonlocally coupled phase oscillators,” Phys. Rev. E 92, 060901 (2015).
${ }^{24}$ M. J. Panaggio and D. M. Abrams, "Chimera states: Coexistence of coherence and incoherence in networks of coupled oscillators," Nonlinearity 28, R67-R87 (2015).

${ }^{25}$ E. A. Martens, M. J. Panaggio, and D. M. Abrams, "Basins of attraction for chimera states: Fast track communication,” New J. Phys. 18, 022002 (2016).

${ }^{26}$ S. Rakshit, B. K. Bera, M. Perc, and D. Ghosh, "Basin stability for chimera states," Sci. Rep. 7, 2412 (2017).

${ }^{27}$ E. Schöll, A. Zakharova, and R. G. Andrzejak, Chimera States in Complex Networks (Frontiers Media S.A., Lausanne, 2020),

${ }^{28} \mathrm{~J}$. C. González-Avella, M. G. Cosenza, and M. San Miguel, "A model for cross-cultural reciprocal interactions through mass media," PLoS One 7, e51035 (2012).

${ }^{29}$ J. C. González-Avella, M. G. Cosenza, and M. San Miguel, "Localized coherence in two interacting populations of social agents," Physica A 399, 24-30 (2014).

${ }^{30}$ A. Reka and A. Barabási, "Statistical mechanics of complex networks," Rev. Mod. Phys. 74, 47 (2002).

${ }^{31} \mathrm{~J}$. C. Harsanyi and R. Selten, A General Theory of Equilibrium Selection in Games (MIT Press, Cambridge, 1988). 